Check for updates

Cite this: RSC Adv., 2019, 9, 6107

Received 1st December 2018

Accepted 2nd February 2019

DOI: 10.1039/c8ra09875a

rsc.li/rsc-advances

\section{A novel nanofiltration membrane with simultaneously enhanced antifouling and antibacterial properties}

\begin{abstract}
Yawei Qi, ${ }^{a}$ Lifang Zhu, ${ }^{\text {ab }}$ Congjie Gao a and Jiangnan Shen (D) *a
A novel nanofiltration membrane prepared by modification of a commercial membrane surface is fabricated using polydopamine (PDA) and hydroxyl propyl trimethyl ammonium chloride chitosan (HACC) mixed with chitosan (CN) and chelated silver (Ag) nanoparticles. The surface chemical composition, cross-sectional morphology, hydrophilicity and surface structure of the prepared membranes were examined by scanning electron microscopy, water contact angle measurements, and atomic force microscopy, respectively. The membrane performance was evaluated in terms of volumetric flux and protein rejection. In addition, the antifouling and antibacterial properties of the membranes were also explored. The results demonstrated that the prepared membranes exhibited an excellent antifouling property due to their three-layer architecture and it provided a special layer to promote the antibacterial property. According to the results, the modified membrane has a significantly improved flux recovery rate (over 96\%). Besides, the antibacterial activity tests showed that the proposed three-layer architecture modification extensively prevented bacterial growth on the membrane surface.
\end{abstract}

\section{Introduction}

A commercial polymeric nanofiltration membrane has a nominal pore size of about $1 \mathrm{~nm}$ and a molecular weight cutoff (MWCO) range of 200 to 1000 . These membranes have been widely used in drinking water production and wastewater treatment., ${ }^{1,2}$ Nanofiltration (NF) is an attractive technology for producing reclaimed water from non-traditional sources such as polluted surface waters and secondary treated effluents, due to its rejection efficiency (selectivity) for high multivalent ions and organic contaminants at a much lower operating pressure than reverse osmosis (RO) technology. ${ }^{3,4}$ Unfortunately, as with all of the membrane filtration processes, an inherent problem with NF is the reduced production efficiency due to fouling of the membrane surface by microorganisms, colloidal materials and dissolved organics. ${ }^{5-7}$ The main factors affecting membrane fouling are roughness and hydrophilicity, as well as the chemical and physical properties of the feed water. ${ }^{8,9}$ The fouling extent of the membrane varies, depending on the structural properties of the membrane..$^{\mathbf{1 0 1 1}}$ In this sense, the incorporation of filler materials in the membrane structure is an appropriate strategy to mitigate the fouling extent. Nano-size metal particulates with enhanced surface areas exhibit superb antimicrobial properties. ${ }^{\mathbf{1 2 - 1 4}}$

${ }^{a}$ Center for Membrane Separation and Water Science \& Technology, Ocean College, Zhejiang University of Technology, Hangzhou 310014, P. R. China.E-mail: shenjn@ zjut.edu.cn

${ }^{b}$ Zhejiang University of Water Resources and Electric Power, Hangzhou 310018, P. R. China
The most common way to improve the hydrophilicity of a membrane surface to mitigate organics deposition onto the membrane surface is the adhesion of a hydrophilic coating to the upper membrane layer. ${ }^{\mathbf{1 5 - 1 7}}$ Commonly used methods are: a hydrophilic polymer is chemically modified and grafted onto the surface of the film by coating or plasma polymerization, so that the hydrophilicity of the film surface is greatly improved. Besides, numerous types of metal or metal oxide nano-size particulates are used to modify the membrane surface, such as silver $(\mathrm{Ag})$ and silver nitrate $\left(\mathrm{AgNO}_{3}\right)$, showing excellent antibacterial properties. ${ }^{\mathbf{1 8 , 1 9}}$

Although these modified methods and materials yielded outstanding characteristics, the instability and bacterial contamination of the modified materials may limit this kind of membrane application in industry. In addition, the regular dispersion of nanoparticles (forming particle aggregates) in the polymeric material and the release of nano-size particulates into the environment are the two main problems in the preparation of an antibacterial membrane. ${ }^{\mathbf{1 3 , 2 0 , 2 1}}$ Thus, the stability of modified layers with enhanced antifouling and antibacterial properties is urgently required in developing polymeric nano-filtration membranes. Unfortunately, membranes with both antifouling and antibacterial properties have been seldom reported. Thus, a "three-layer" modified structure was designed using poly-dopamine (PDA), with self-polymerization in the presence of oxygen and hydroxyl propyl trimethyl ammonium chloride chitosan (HACC) mixed with chitosan (CN) and chelated silver (Ag) nanoparticles. 
Dopamine has attracted much attention due to its strong adhesion and antifouling property on the surface of materials. $^{\mathbf{1 0 , 2 2}}$ Based on the strong adhesion to solid material surfaces and intrinsic ability of easy secondary treatment of dopamine, a large number of methods have been proposed for the surface modification of solid materials..$^{23-26}$ In addition, due to the presence of amino and hydroxyl functional groups on dopamine, researchers used dopamine as an intermediate for further reaction; for example, Prokopios G. et al. used the Michael addition reaction to successfully make dopamine derivatives. ${ }^{27}$ Furthermore, the antifouling properties of a membrane surface can be enhanced. HACC is a non-polluting material and it easily solidifies into a methyl silicone membrane..$^{28-31}$ Moreover, the chitosan derivatives containing quaternary ammonium salt with a large number of amino groups favor the formation of water insoluble complexes with anionic polyelectrolytes and chelate metal ions. ${ }^{32,33}$ The chelation of chitosan quaternary ammonium salt with nano-silver (HACC\&Ag) was achieved by a green in situ formation method: HACC solution was mixed with dissolved chitosan, then chelated with silver nitrate, and acetic acid was used as a reducing agent, as described by Kumar-Krishnan et al. ${ }^{\mathbf{3 4}}$

In this work, a "three-layer" stable structure is mainly determined by the presence of PDA in the inner most and upper layer. The inner most layer is used to promote the formation of adherent polymer coatings on a commercial membrane surface and provide coordinate bonds between the two inner layers, leading to the formation of a crosslinked structure with a middle layer (formed by HACC-CN\&Ag). This middle layer is designed to prevent bacterial contamination. An additional uppermost PDA layer is deposited onto the middle layer in order to guarantee the stability of the HACC-CN\&Ag layer. The antibacterial property of the middle layer could be lost by the effect of the interacting or adhered fouling compounds. Therefore, the PDA-(HACC-CN\&Ag)-PDA modification structure was designed and coated on a commercial nanofiltration membrane surface. Simultaneously, experiments were performed to evaluate the antifouling property and antibacterial activity of the modified commercial NF membranes using sodium alginate (SA) and humic acid (HA) as membrane foulants and colon bacillus as representative bacteria, respectively (Fig. 1).

\section{Experimental}

\subsection{Materials and chemicals}

NF-90 and NF-270 commercial membranes were used as support materials to fabricate the advanced NF membranes in this investigation. 2-Hydroxypropyltrimethyl ammonium chloride chitosan (HACC, >99.9\%), dopamine hydrochloride (PDA, $>98 \%)$, acetic acid (dilute acetic acid, $1 \%)$ silver nitrate $\left(\mathrm{AgNO}_{3}\right.$, $>99 \%$ ), humic acid (HA, >90\%), sodium alginate (SA, >90\%), chitosan (>95\%) and all other chemicals were obtained from Aladdin Industrial Co. (Shanghai, China).

\subsection{Membrane preparation}

The commercial NF-90 and NF-270 membranes were firstly rinsed with deionized water to clean them and then stored in $0.6 \mathrm{M} \mathrm{NaCl}$ solution for $12 \mathrm{~h}$.

First, $0.30 \mathrm{~g}$ of dopamine hydrochloride was immersed in $150 \mathrm{~mL}$ of a $20 \mathrm{~mm}$ trihydrochloric acid buffer $(\mathrm{pH}=8.6)$. The solution and membrane were then added to a laboratory setup with a $110 \mathrm{rpm}$ stir bar. The membrane surface color changed from clear to dark brown when the solution was in contact with oxygen. The reaction route is shown in Fig. 2 . After $12 \mathrm{~h}$, the

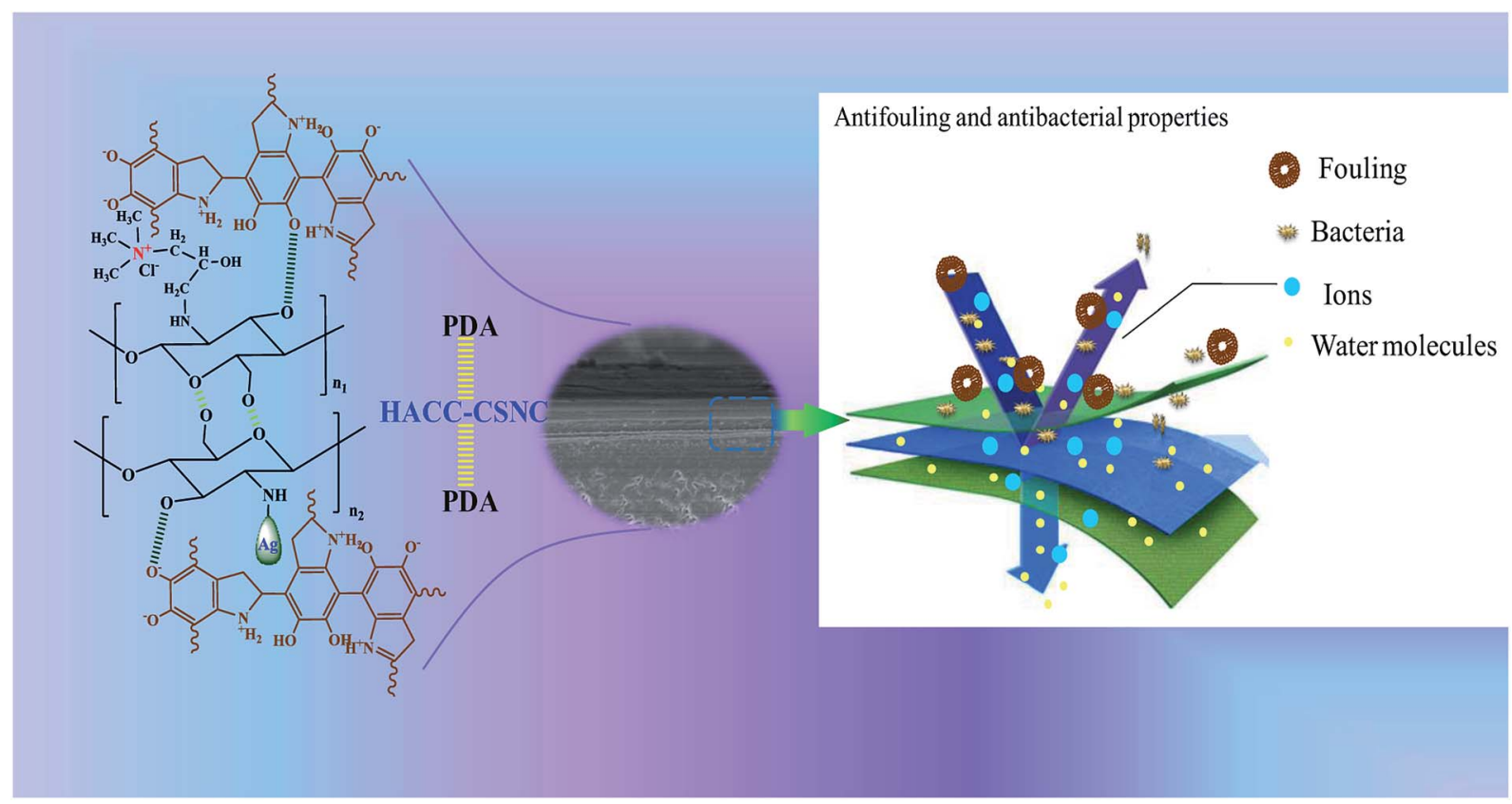

Fig. 1 Diagram of the three-layer structure with antifouling and antibacterial effects. 

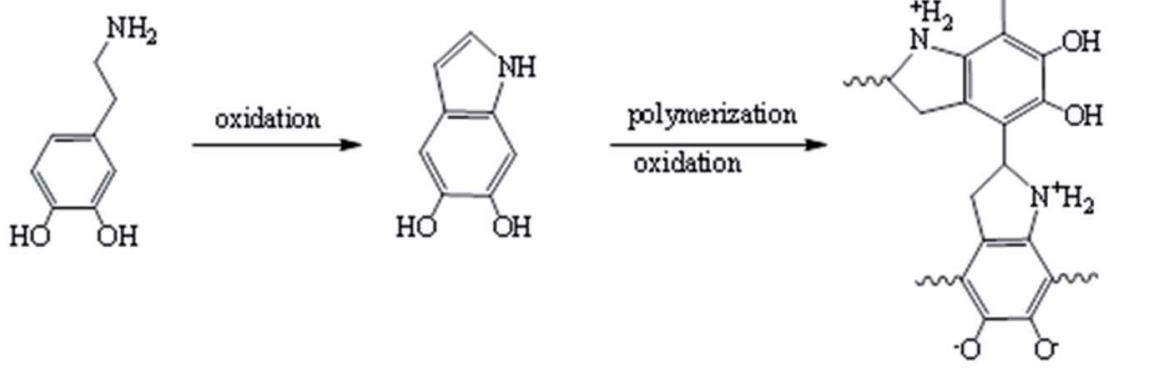

Dopamine solution

5,6-dihydroxyindole

Polydopamine (PDA)

Fig. 2 Reaction route for the poly dopamine polymerization.

formed particulate impurities on the surface of the added PDA layer were rinsed with deionized water.

The second layer was formed by the addition of HACC\&Ag (HACC $0.5 \mathrm{~g} \mathrm{~L}^{-1}$ solution with dissolved chitosan $\left(0.5 \mathrm{~g} \mathrm{~L}^{-1}\right)$ chelated with silver nitrate $\left(0.2 \mathrm{mg} \mathrm{L}^{-1}\right) ; 1 \%$ acetic acid was used as a reducing agent ${ }^{34}$ ). This mixed solution (HACC\&Ag, see the structure in Fig. 3 and 4) was poured into the experimental setup with $150 \mathrm{rpm}$ stirring rods. $12 \mathrm{~h}$ later, the film was also rinsed with deionized water.

The last layer was made using the same procedure as the first PDA layer within $12 \mathrm{~h}$ from dopamine hydrochloride. The film was thoroughly rinsed with deionized water between the coating steps. Finally, the modified membrane was immersed and stored in deionized water.

\subsection{Membrane characterization}

The surface morphology of the NF-90 and NF-270 membranes was observed by using scanning electron microscopy (SEM) (S5700 , China) and atomic force microscopy (AFM) (Nanoscope V,
United Kingdom), respectively. The water contact angle of the films was measured on a goniometer by a fixed-axis drop method. The membrane was cut into two pieces $(1 \mathrm{~cm} \times 2 \mathrm{~cm})$ and placed on the surface of the mold, which was filled with $0.6 \mathrm{~mol}$ of potassium chloride solution (NaCl-aq) for testing.

\subsection{Separation performance of membranes}

The filtration performance of the NF membranes before and after modification was evaluated by using a cross-flow filtration system. The concentration of the feed solutions $(200 \mathrm{ppm} \mathrm{NaCl}$ and $500 \mathrm{ppm} \mathrm{Na}_{2} \mathrm{SO}_{4}$ ) was prepared by dissolving in deionized water and using $\mathrm{NF}$ membrane as a raw material to evaluate the rejection performance after NF membrane modification under 10 bar pressure at $25^{\circ} \mathrm{C}$. All of the experiments were carried out many times. Pure water flux $(J)$ and salt rejection rate $(R)$ were calculated using eqn (1) and (2), respectively.

$$
J=\frac{V}{A \times \Delta t}
$$

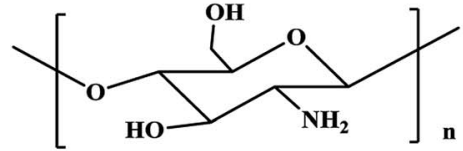

(a)

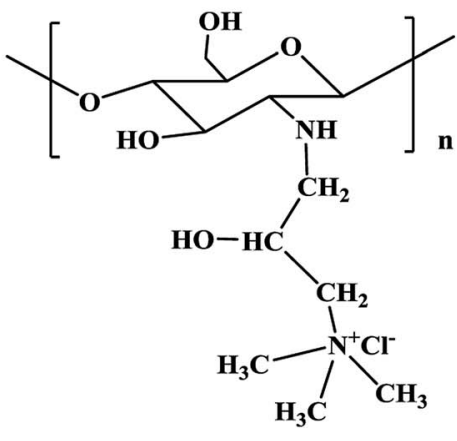

(b)

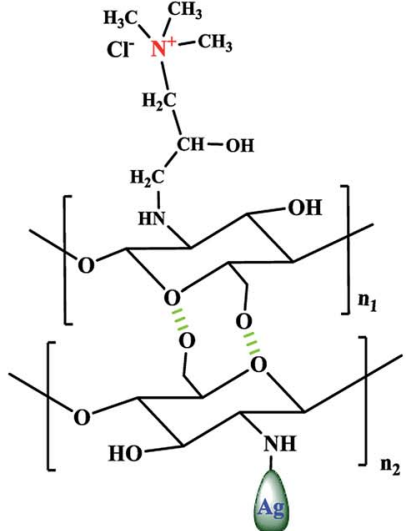

(c)

Fig. 3 The chemical structures of chitosan (a) and 2-hydroxypropyltrimethyl ammonium chloride chitosan (b). The chemical structures of HACC mixed with chitosan and chelated with silver nanoparticles (c). 


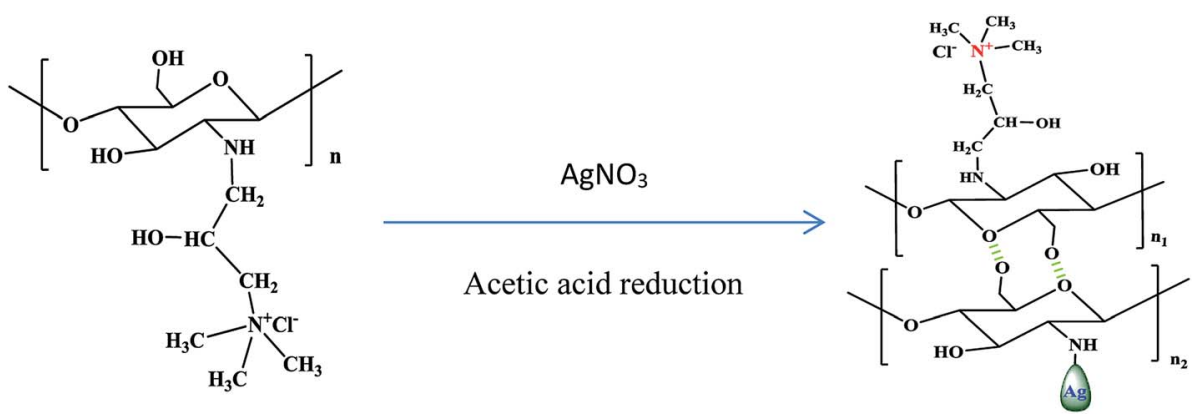

Fig. 4 Chelation of chitosan and silver nanoparticles.

where $V$ is the volume collected from the permeate (L), $A$ represents the area of water passing through the membrane $\left(\mathrm{m}^{2}\right)$, and $\Delta t$ refers to the time required to collect a certain amount of water $(\mathrm{h})$.

$$
R(\%)=\left\{1-\frac{C_{\mathrm{p}}}{C_{\mathrm{f}}}\right\} \times 100
$$

where $C_{\mathrm{p}}\left(\mathrm{mg} \mathrm{L}^{-1}\right)$ and $C_{\mathrm{f}}\left(\mathrm{mg} \mathrm{L}^{-1}\right)$ represent permeate and feed concentrations, respectively.

\subsection{Antifouling performance measurements}

The antifouling performance of the membranes was evaluated by using an over-flow filtration test on a single solute aqueous solution containing SA and HA model foulants. The fouling tests were carried out using the circulation model at room temperature. The membrane samples in the filtered cells were pressurized with deionized water at a pressure of 10 bar for about $2 \mathrm{~h}$ to ensure stable membrane flux before the soil test. The concentration of both SA and HA solutes in the feed solution was $200 \mathrm{mg} \mathrm{L}^{-1}$ and the permeation flux with time was measured periodically until a steady state value was reached. The tested membrane was rinsed under a decanter with deionized water for about $50 \mathrm{~min}$ to remove the fouling molecules deposited on the membrane surface and the water flux was re-measured under the same test trans membrane pressure. The fouling behavior of the membranes was investigated using a membrane-dependent normalized flux profile.

\subsection{Antibacterial performance measurements}

The antibacterial properties of the tested membranes were evaluated by using LB solid medium. ${ }^{7,8,14,32}$ Specifically, these experiments were performed by using LB solid medium and $E$. coli DH5a, sterilization scissors for the diaphragm cutting $(1 \times$ $1 \mathrm{~cm}$ in size), and using the absorbance of $600 \mathrm{~nm}$ light. The protocol used is described as follows. Extract 100 microliters of E. coli DH5a from a suspension into LB solid medium and apply evenly with a coating stick. The diaphragm is lightly attached to the surface of the culture medium (the front of the diaphragm is in contact with the medium surface). Finally, the LB solid plate is inverted (as shown in Fig. 14), and the samples are put into incubators for $24 \mathrm{~h}$ at $37{ }^{\circ} \mathrm{C}$. Observe the samples: if the diaphragm surface is sterile and there is no growth, this indicates that it has antibacterial properties; otherwise, the diaphragm has no antibacterial effect. ${ }^{32}$

\section{Results and discussion}

\subsection{Physico-chemical membrane characterization}

The effect of the three-layer modification architecture on the surface of the modified membrane was observed by using scanning electron microscopy. Fig. 5 shows the surface topography and cross-section of the NF-90 film. The unmodified NF-90 film (Fig. 5a) shows a rough surface. Thus, fouling materials may block the membrane surface during the filtration process. In addition, Fig. $5 \mathrm{~b}$ shows a crosssection image of the unmodified NF-90 membrane, suggesting a homogeneous morphology for its surface. For the modified NF-90 membrane (Fig. 5c), as a result of the architecture layer modification, the rough morphology disappeared and a rather smoother surface appeared. In Fig. 5d, the membrane modified (added) layer can be clearly distinguished from the cross-section image of the film and the thickness of the modified layers is about $2.0 \mu \mathrm{m}$. Besides, the surface and cross-section images of the unmodified and modified NF-270 membranes are shown in Fig. 6. Fig. 6a displays the smooth surface of the unmodified NF-270 membrane and the homogeneous nanofiltration layer can be observed in Fig. 6b. The morphology of the membrane surface was altered by the three-layer modification architecture applied on the membrane surface, as shown in Fig. 6c and $\mathrm{d}$. It is notable that the rougher surface of the modified NF-270 membrane and cross-sections exhibited the obviously distinguishable modification layer. Hence, an improved performance of the modified NF membranes is expected as a result of the addition to the membrane surface of the layer with the PDA-(HACC-CN\&Ag)-PDA modification architecture.

In the 3D AFM images in Fig. 7, the surface roughness of the unmodified (Fig. 7a), PDA modified (Fig. 7b) and threelayer modified (Fig. 7c) NF-90 membranes is displayed. Besides, the surface roughness of the unmodified (Fig. 8a), PDA modified (Fig. 8b) and three-layer modified (Fig. 8c) NF270 membranes is shown in the 3D AFM images in Fig. 8. The obtained values of roughness for the modified NF-90 membranes by PDA and the three-layer architecture were 

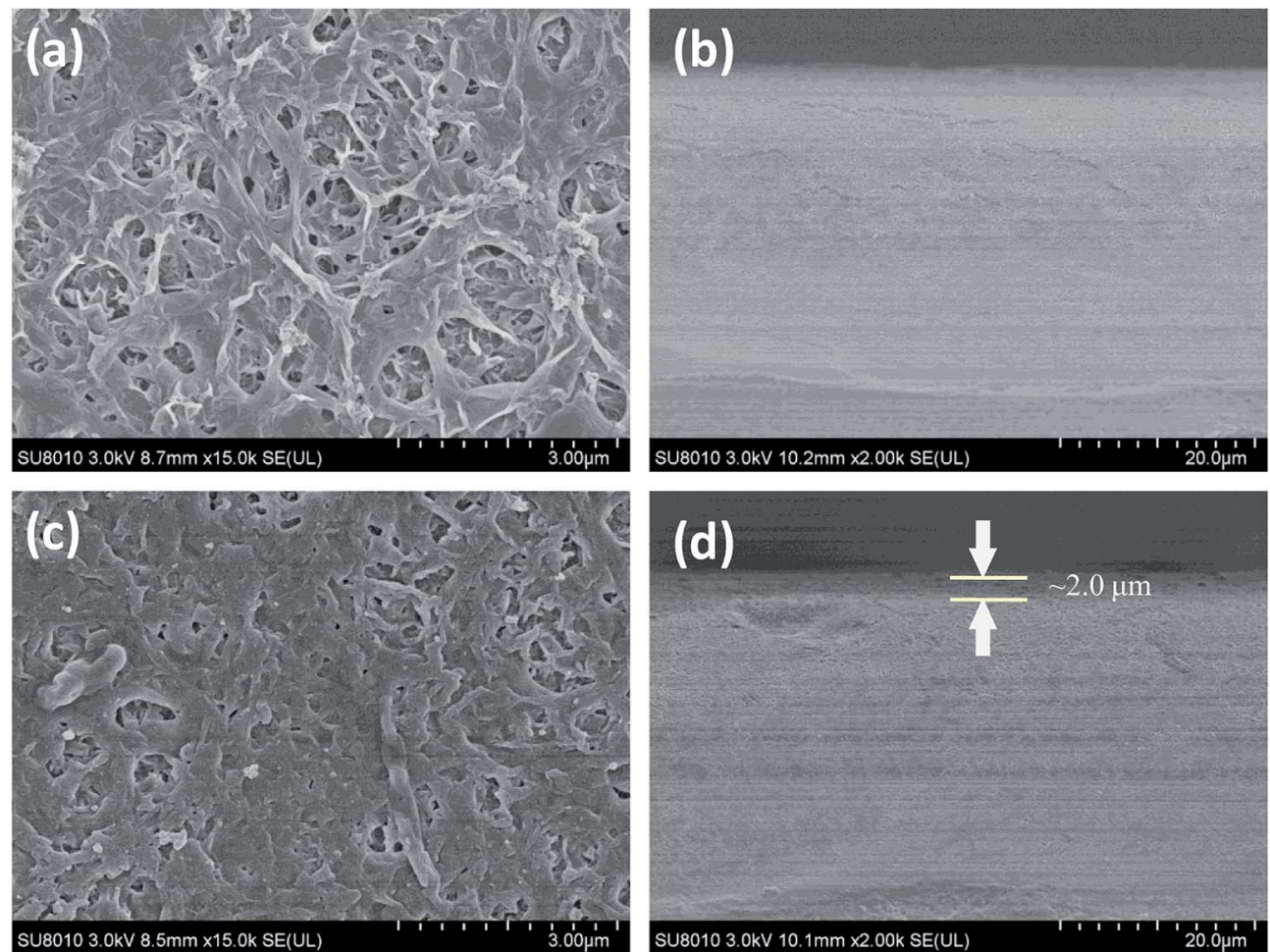

Fig. 5 Unmodified NF-90 membrane surface (a) and cross-sectional SEM micrograph (b); three layer modified NF-90 membrane surface (c) and cross-sectional SEM micrograph (d).

found to be $87.0 \mathrm{~nm}$ and $108.0 \mathrm{~nm}$, respectively; both values are higher than that of the unmodified NF-90 membranes $(73.5 \mathrm{~nm})$. In the case of the NF-270 membrane, the surface roughness slightly increased from $9.4 \mathrm{~nm}$ to $10.1 \mathrm{~nm}$ and 13.2 $\mathrm{nm}$ due to the proposed modifications by PDA and the three-layer architecture, respectively.
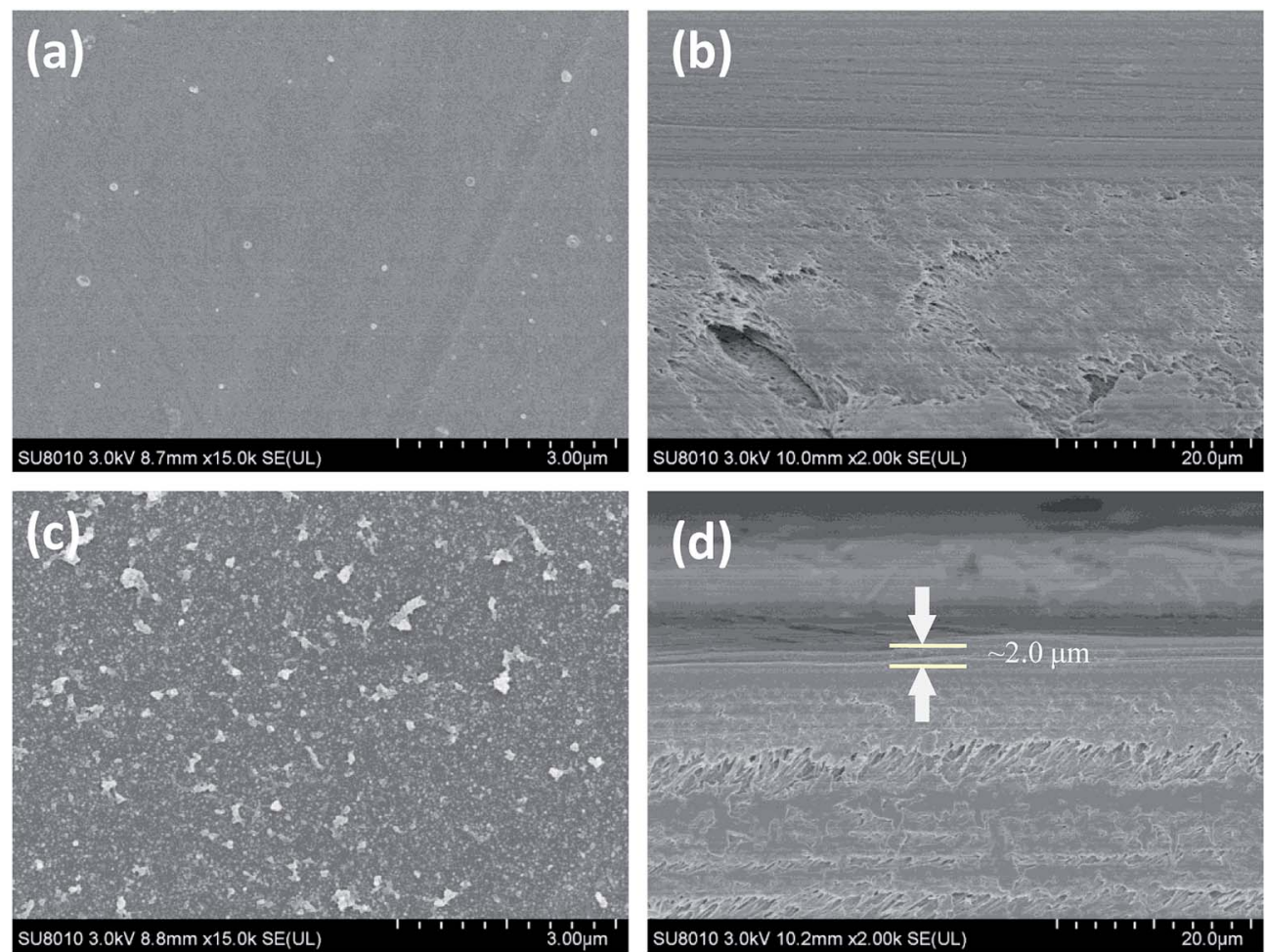

Fig. 6 Unmodified NF-270 membrane surface (a) and cross-sectional SEM micrograph (b); three layer modified NF-270 membrane surface (c) and cross-sectional SEM micrograph (d). 

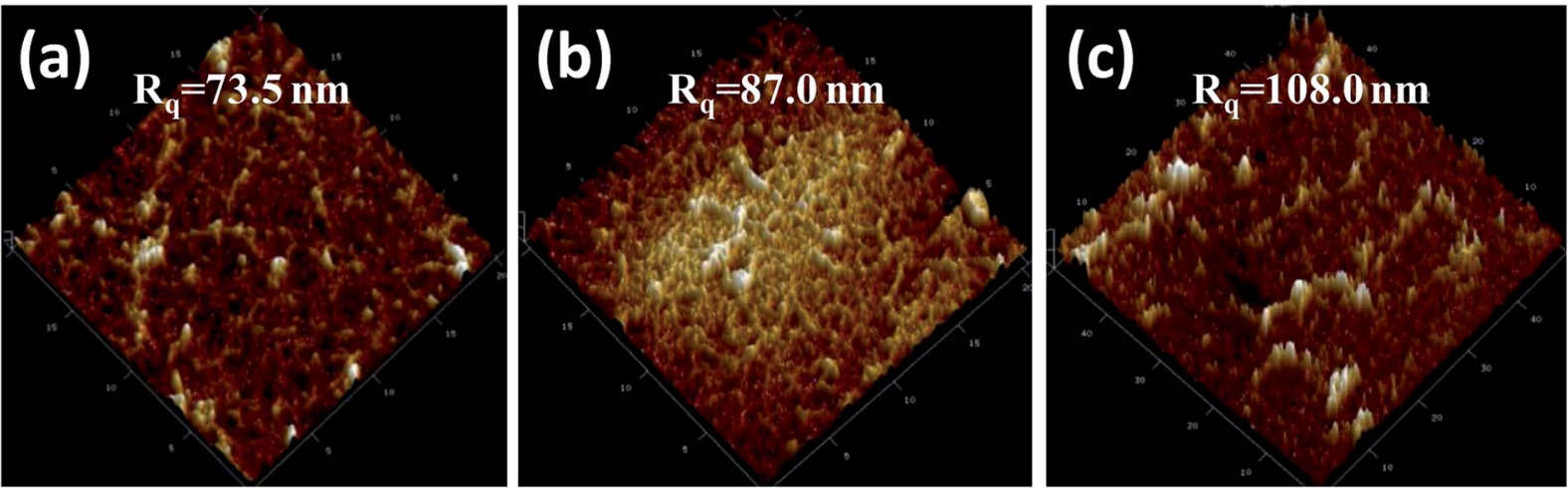

Fig. 7 AFM images of the surface of the unmodified (a), PDA modified (b) and three layer modified NF-90 membrane surface (c).
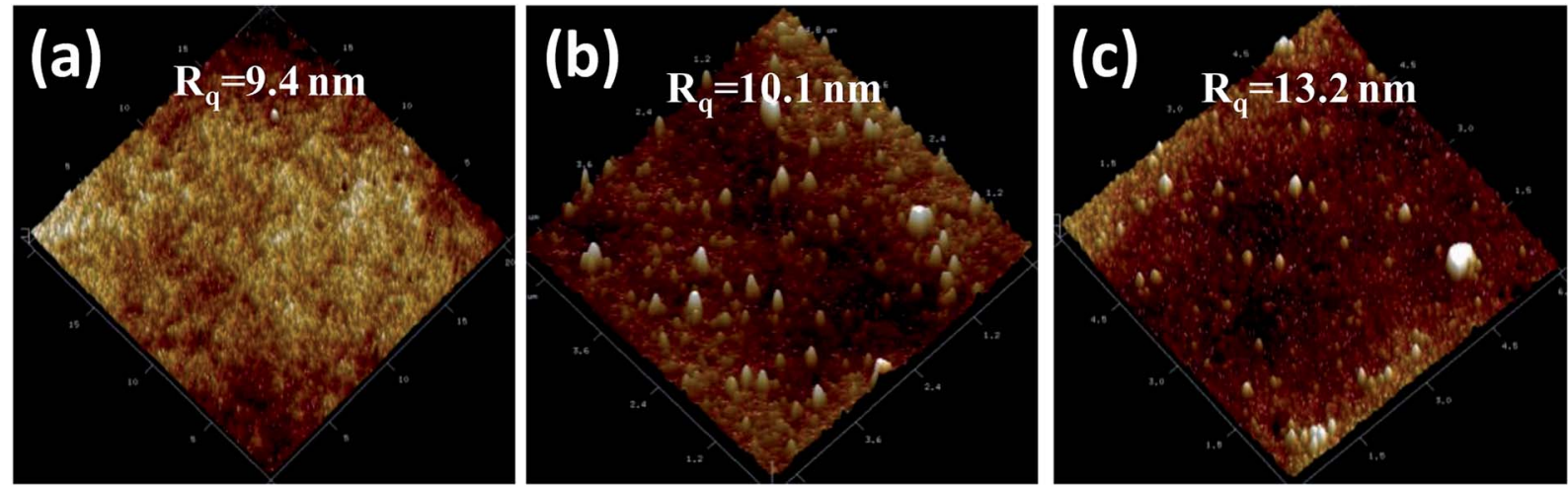

Fig. 8 AFM images of the surface of the unmodified (a), PDA modified (b) and three layer modified NF-270 membrane surface (c).

The contact angle measurements of the NF-90 and NF-270 membranes are shown in Fig. 9. Due to the great difference in hydrophilicity between the two commercial membranes, the hydrophilicity of the modified membranes exhibits different trends. For NF-90, the original membrane is less hydrophilic. As result of PDA deposition, a large number of hydrophilic amino groups and hydroxyl groups are added to the surface of the commercial membrane, increasing the hydrophilic character of the modified membrane. However, further modification involving the formation of the three layer structure did not increase the surface hydrophilicity, rather it slightly decreases. This effect could be associated with the unevenness of the modified surface.

On the contrary, for NF-270, the contact angle increased. It is important to point out that the NF-270 membrane is considered as a loose NF membrane because of its high (a)

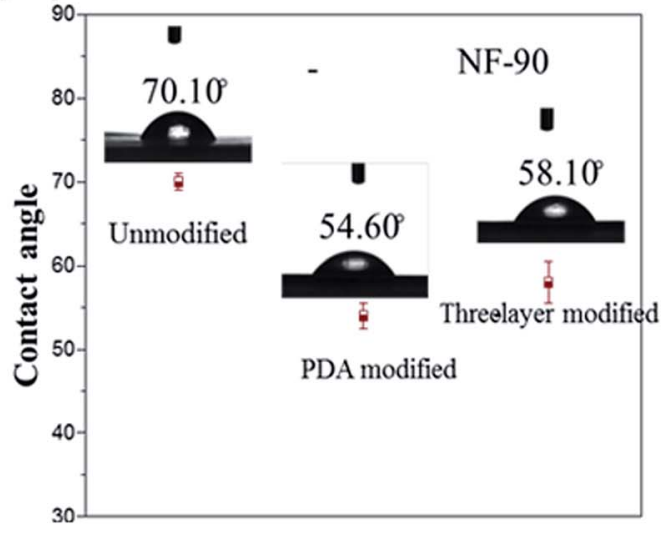

Membranes' type (b)

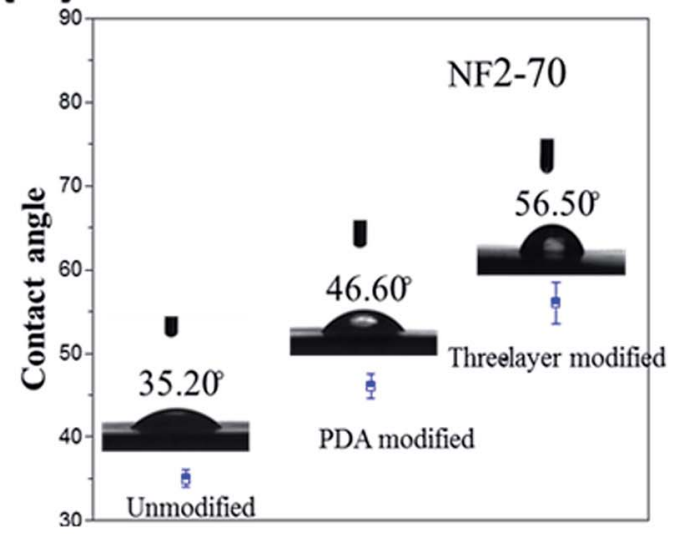

Membranes' type

Fig. 9 Contact angle of the NF-90 membrane surface (a) and NF-270 membrane surface (b). 
(a)

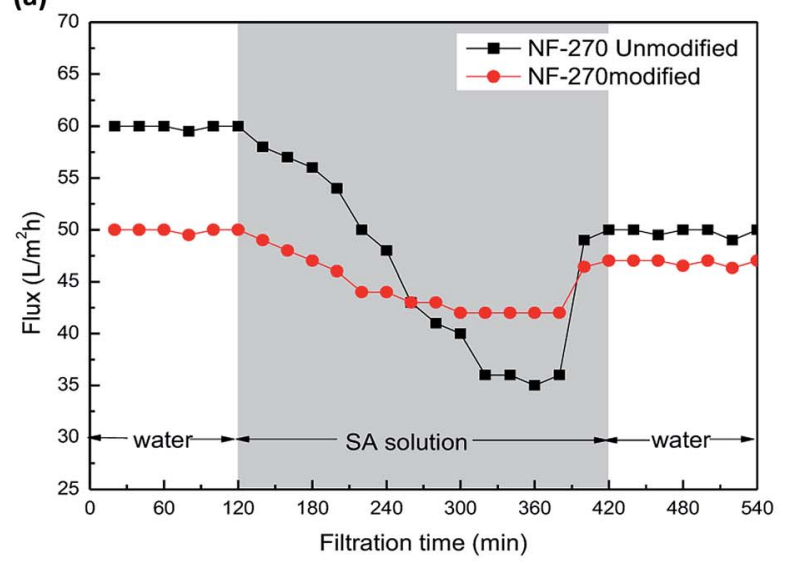

(b)

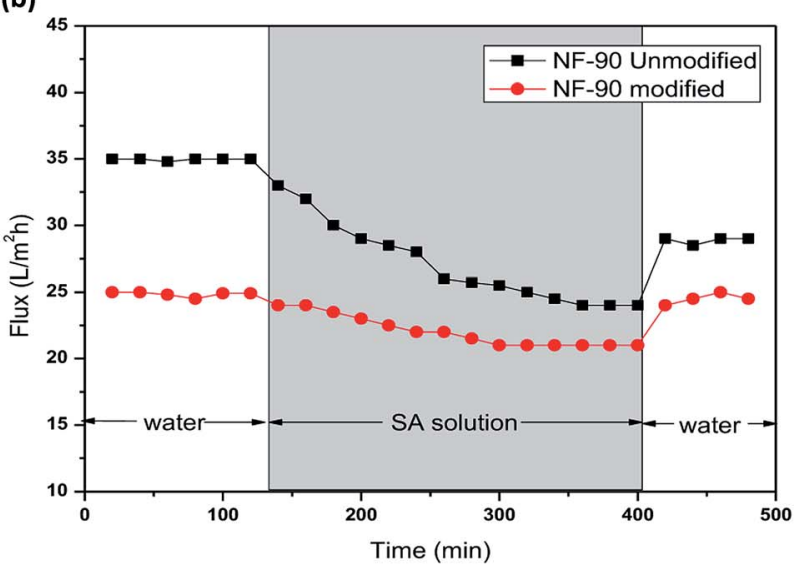

Fig. 10 Membrane filtration performance of the NF-90 membranes and antifouling behavior in the presence of humic acid (a) and sodium alginate (b).

porosity in comparison with a tight NF membrane, such as the NF-90 membrane. This effect can be explained in terms of a surface pore size decrease provoked by the deposition of the additional layers onto the membrane surface. This explanation can be supported by the similar final values observed for both membranes as a result of the same surface modification. This also indicates that the modification of the film was successful.

\subsection{Membrane filtration performance and antifouling behavior}

In these experiments, humic acid and sodium alginate, which are common nanofiltration surface fouling substances in water treatment processes, were selected to assess the antifouling potential of the modified membranes. As shown in Fig. 10, the porosity of the modified membranes was reduced, leading to a decrease of water permeation flux. After sodium alginate and humic acid solution filtration, the water flux declined and finally became stable. Then, the initial water flux was recovered after a cleaning protocol with deionized water.
As can be seen from Fig. 10 and 11, the recovery of the modified membrane is higher than that of the original commercial samples, suggesting that irreversible organic fouling of the membrane can be reduced by the surface modification proposed in this investigation.

At the same time, SEM images of the modified and unmodified nanofiltration membrane surfaces were taken to evaluate the fouling behavior of the prepared membranes. Fig. 12a and $\mathrm{b}$ show the foulant adsorption onto the unmodified NF-90 membrane surface. However, the membrane surface modified by the three-layer architecture exhibits a clean surface without obvious dirt deposition, as shown in Fig. 12c and d.

In addition, the NF-270 membrane, which has a smooth surface, can also be fouled by sodium alginate and humic acid (respectively shown in Fig. 13a and b). However, for the improved film, after the same treatment, the surface became smoother and cleaner, as shown in Fig. 13c and d. Thus, it can be concluded that the antifouling properties of NF membranes can be improved by the three-layer architecture modification used in this study.
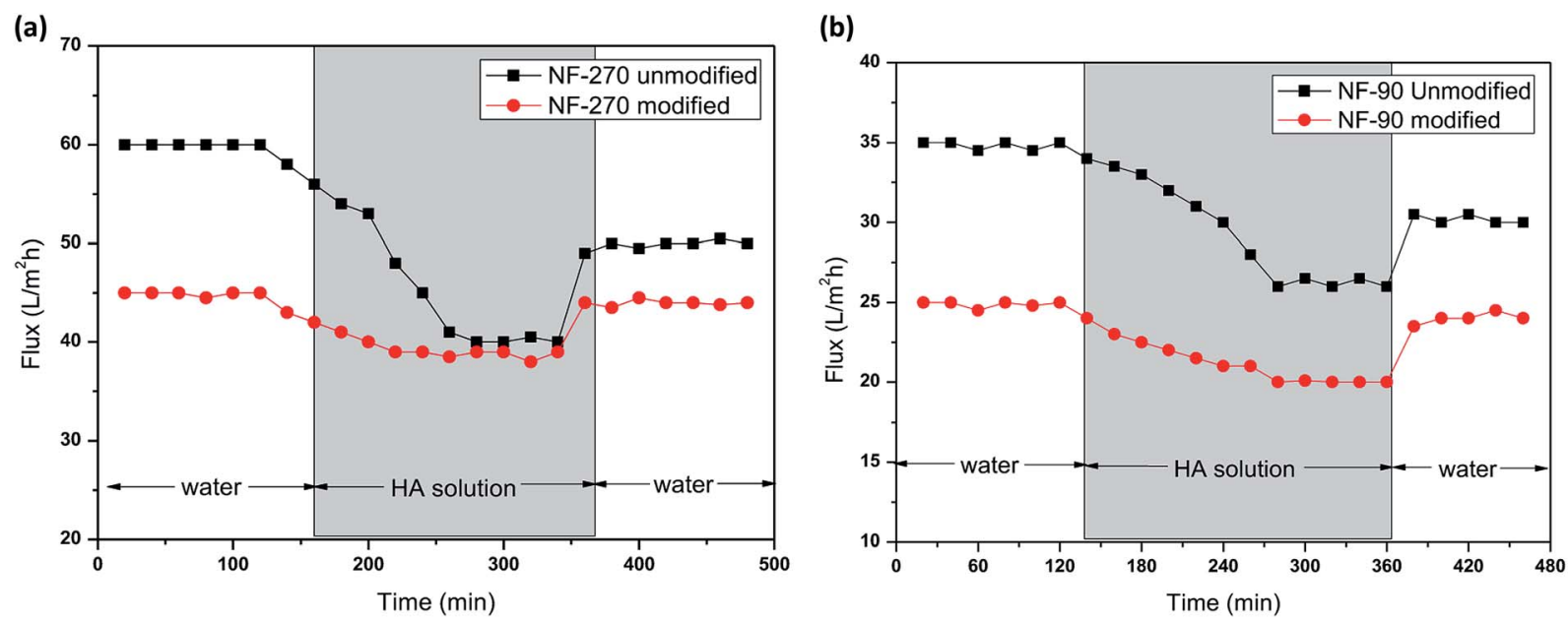

Fig. 11 Membrane filtration performance of NF-270 and antifouling behavior in the presence of humic acid (a) and sodium alginate (b). 

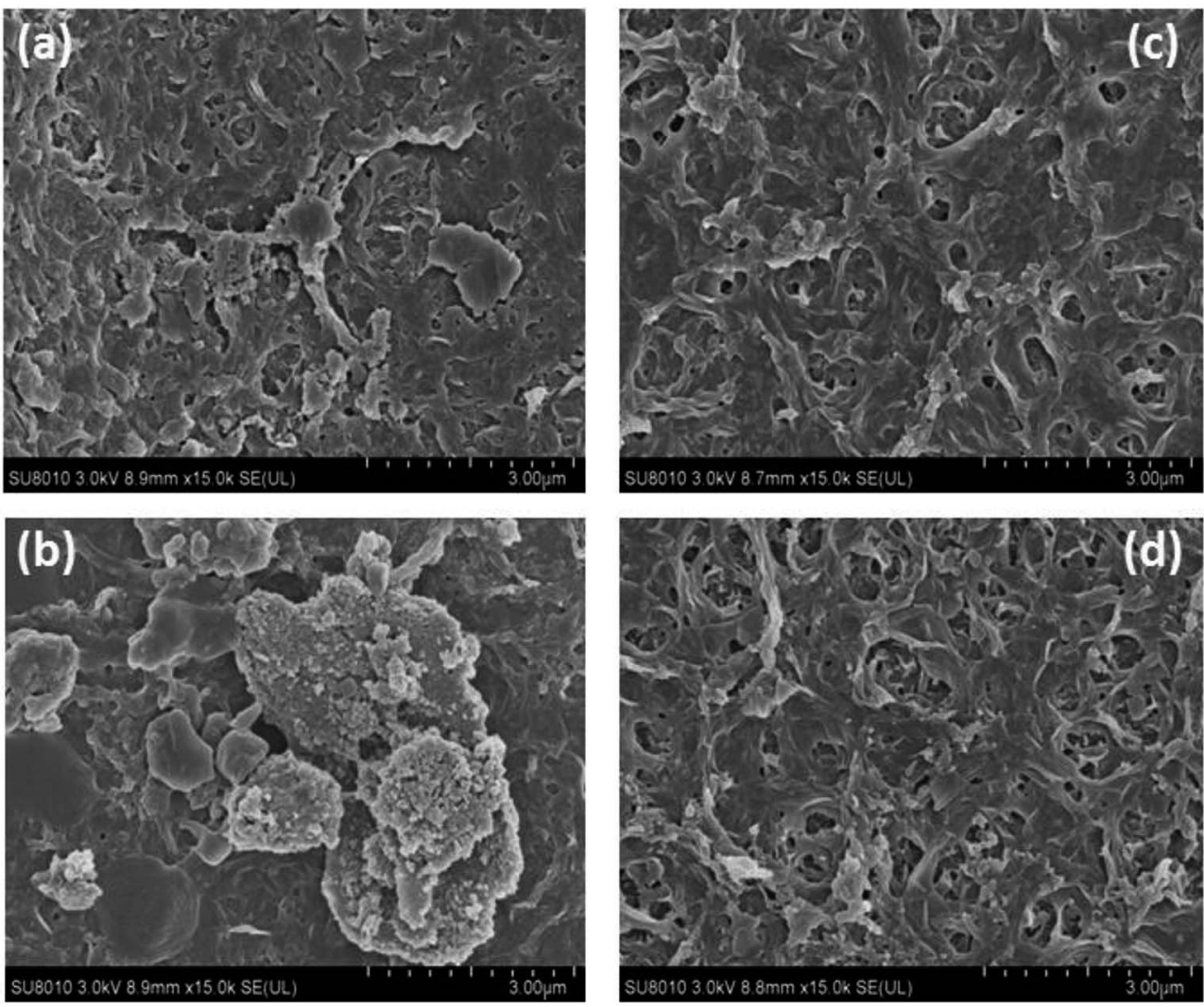

Fig. 12 The antifouling behavior of the NF-90 membranes: (a) unmodified membrane fouled by sodium alginate; (c) modified membrane fouled by sodium alginate; (b) unmodified membrane fouled by humic acid; (d) modified membrane fouled by humic acid.
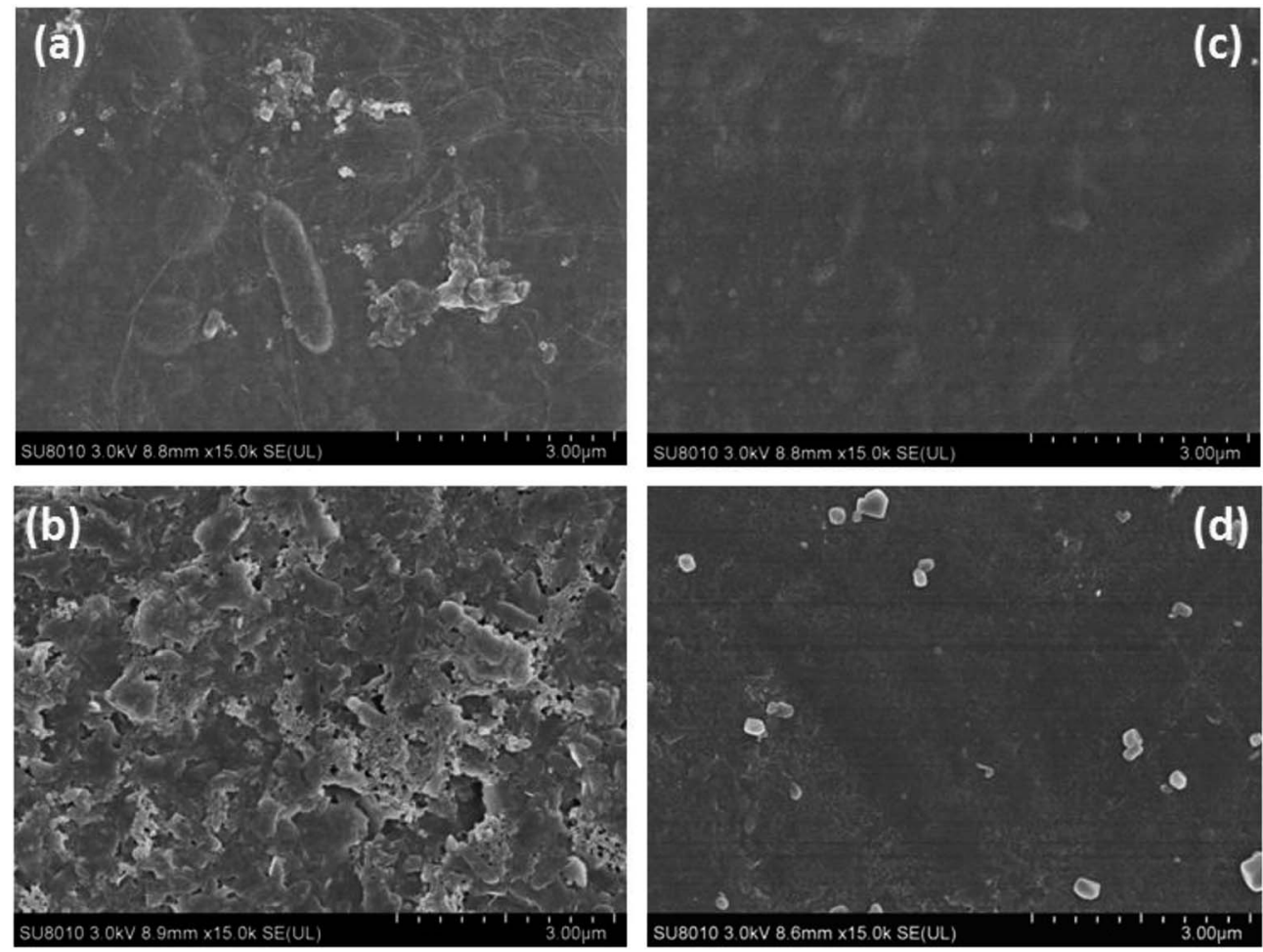

Fig. 13 The antifouling behavior of NF-270: (a) unmodified membrane fouled by sodium alginate; (c) modified membrane fouled by sodium alginate; (b) unmodified membrane fouled by humic acid; (d) modified membrane fouled by humic acid. 

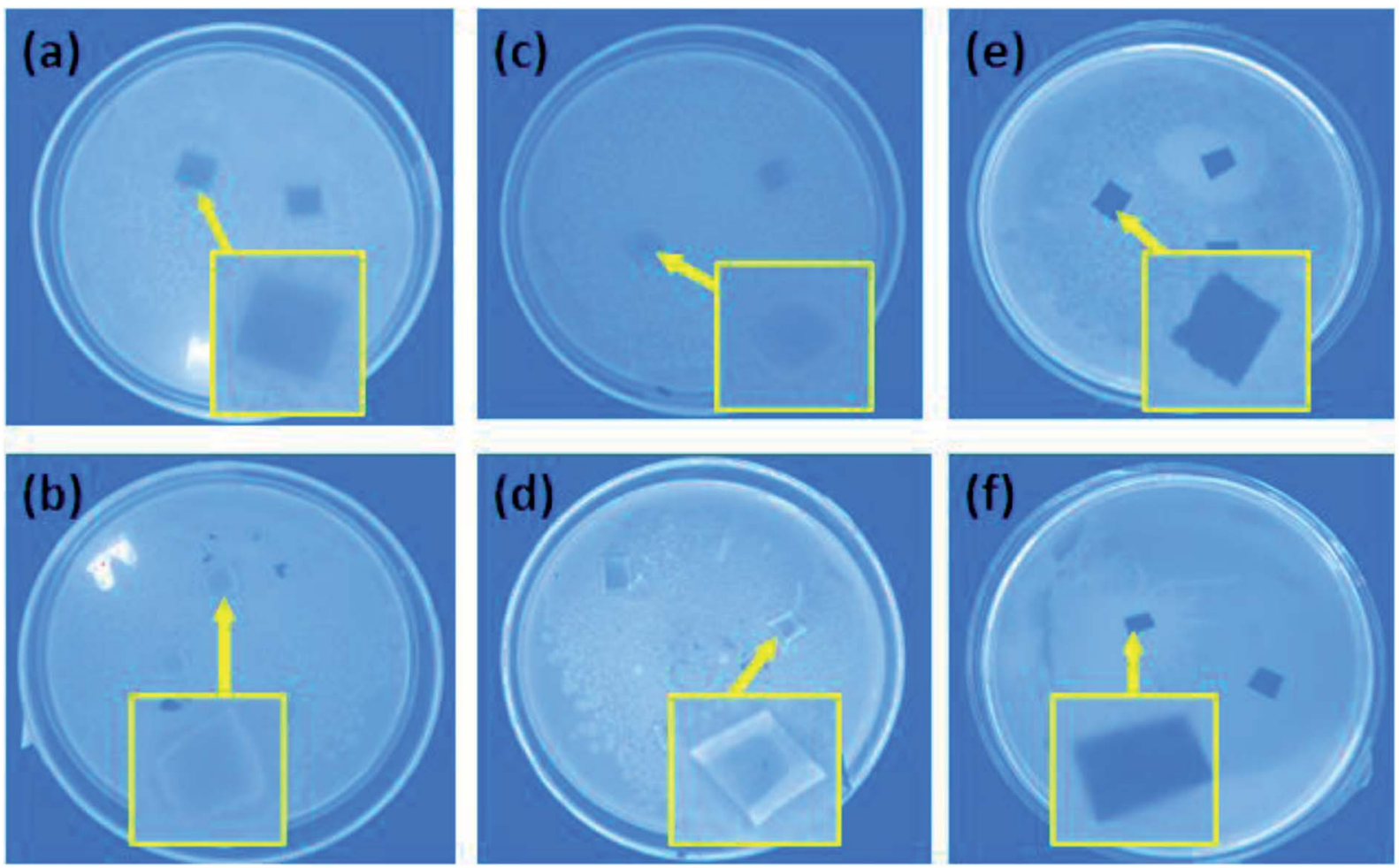

Fig. 14 The antimicrobial behavior in LB solid medium: (a) unmodified, (c) PDA modified, and (e) three-layer modified NF-90; (b) unmodified, (d) PDA modified, and (f) three-layer modified NF-270.

\subsection{Antimicrobial behavior}

Escherichia coli were used as model bacteria to evaluate the antibacterial activity resulting from the modification of the NF270 and NF-90 commercial membranes. The images in Fig. 14 show the antimicrobial behavior of the unmodified, PDA modified and three-layer architecture modified nanofiltration membranes. The unmodified NF-270 and NF-90 membranes were both contaminated by the model bacteria (shown in Fig. 14a and b) after $48 \mathrm{~h}$ of experimental time, suggesting the reduced antibacterial properties of the commercial membranes.

As shown in Fig. 14c and d, the antibacterial activity of the membranes slightly increased as a result of the first PDA layer deposition on their surfaces. The bacterial growth was reduced under the effect of the additional active functional groups of PDA. However, as a result of the further membrane surface modification by the three-layer architecture strategy, as shown in Fig. 14e and f, no bacterial contamination on the membrane surface could be observed. Thus, this behavior illustrates that the three-layer architecture enables excellent antimicrobial activity for the modified NF membranes.

\section{Conclusions}

In this study, commercial NF-270 and NF-90 membranes were modified by poly dopamine and by a "three-layer" modification architecture, PDA-(HACC-CN\&Ag)-PDA, on the surface of the membranes. The modified membranes were verified by electron microscopy (SEM), atomic microscopy (AFM) and contact angle measurement.
The anti-fouling performance of the membranes was evaluated using sodium alginate and humic acid as fouling raw materials, and the anti-fouling performance of the modified membranes compared with the original membranes was evaluated by observing the change of water flux. The results showed that the flux decline rate of the modified membranes was significantly lower than that of the original membranes, and the flux recovery rate was significantly higher than that of the original membranes. The results show that the modified membranes have better anti-fouling performance and this was further confirmed by electron microscopy (SEM). E. coli were used as the strain to evaluate the antibacterial properties of the membranes. The results showed that the antibacterial effect of the modified membranes was significantly better than that of the original membranes. Finally, this study provides a new modification method for membranes and proves that antibacterial and anti-pollution effects were achieved successfully.

\section{Conflicts of interest}

There are no conflicts to declare.

\section{Acknowledgements}

The research was supported by the National High Technology Research and Development Program 863 (No. 2015AA030502), the Public Welfare Project of the Science and Technology Committee of Zhejiang Province (No. 2013C31038), and the 
Natural Science Foundation of Zhejiang Province (No. Y16B060039).

\section{References}

$1 \mathrm{~J}$. Yin and B. Deng, Polymer-matrix nanocomposite membranes for water treatment, J. Membr. Sci., 2015, 479, 256-275.

2 J. Zhu, M. Tian, Y. Zhang, H. Zhang and J. Liu, Fabrication of a novel "loose" nanofiltration membrane by facile blending with Chitosan-Montmorillonite nanosheets for dyes purification, Chem. Eng. J., 2015, 265, 184-193.

3 A. K. Shukla, J. Alam, M. Alhoshan, L. A. Dass and M. R. Muthumareeswaran, Development of a nanocomposite ultrafiltration membrane based on polyphenylsulfone blended with graphene oxide, Sci. Rep., 2017, 7, 41976.

4 R. Pang, X. Li, J. Li, Z. Lu, X. Sun and L. Wang, Preparation and characterization of $\mathrm{ZrO}_{2} / \mathrm{PES}$ hybrid ultrafiltration membrane with uniform $\mathrm{ZrO}_{2}$ nanoparticles, Desalination, 2014, 332, 60-66.

5 S. Liu, F. Fang, J. Wu and K. Zhang, The anti-biofouling properties of thin-film composite nanofiltration membranes grafted with biogenic silver nanoparticles, Desalination, 2015, 375, 121-128.

6 A. Mollahosseini and A. Rahimpour, Interfacially polymerized thin film nanofiltration membranes on $\mathrm{TiO}_{2}$ coated polysulfone substrate, J. Ind. Eng. Chem., 2014, 20, 1261-1268.

7 Y. Xie, C. Tang, Z. Wang, Y. Xu, W. Zhao, S. Sun and C. Zhao, Co-deposition towards mussel-inspired antifouling and antibacterial membranes by using zwitterionic polymers and silver nanoparticles, J. Mater. Chem. B, 2017, 5, 71867193.

8 M. Wasim, A. Sabir, M. Shafiq, A. Islam, M. Azam and T. Jamil, Mixed matrix membranes: Two step process modified with electrospun (carboxy methylcellulose sodium salt/sepiolite) fibers for nanofiltration, J. Ind. Eng. Chem., 2017, 50, 172-182.

9 H. Li, W. Shi, Q. Du, R. Zhou, H. Zhang and X. Qin, Improved separation and antifouling properties of thin-film composite nanofiltration membrane by the incorporation of cGO, Appl. Surf. Sci., 2017, 407, 260-275.

10 Y. Zhao, H. Liu, K. Tang, Y. Jin, J. Pan, B. V. der Bruggen, J. Shen and C. Gao, Mimicking the cell membrane: bioinspired simultaneous functions with monovalent anion selectivity and antifouling properties of anion exchange membrane, Sci. Rep., 2016, 6, 37285.

11 H. Song, H. Yu, L. Zhu, L. Xue, D. Wu and H. Chen, Durable hydrophilic surface modification for PTFE hollow fiber membranes, React. Funct. Polym., 2017, 114, 110-117.

12 X. Hu, X. Lin, H. Zhao, Z. Chen, J. Yang, F. Li, C. Liu and F. Tian, Surface functionalization of polyethersulfone membrane with quaternary ammonium salts for contactactive antibacterial and anti-biofouling properties, Materials, 2016, 9, 376.
13 X.-D. Weng, Y.-L. Ji, R. Ma, F.-Y. Zhao, Q.-F. An and C.-J. Gao, Superhydrophilic and antibacterial zwitterionic polyamide nanofiltration membranes for antibiotics separation, $J$. Membr. Sci., 2016, 510, 122-130.

14 P. Kaner, D. J. Johnson, E. Seker, N. Hilal and S. A. Altinkaya, Layer-by-layer surface modification of polyethersulfone membranes using polyelectrolytes and $\mathrm{AgCl} / \mathrm{TiO}_{2}$ xerogels, J. Membr. Sci., 2015, 493, 807-819.

15 Y. Liu, R. Wei, O. Lin, W. Zhang, Y. Du, C. Wang and C. Zhang, Enhanced hydrophilic and antipollution properties of PES membrane by anchoring $\mathrm{SiO}_{2} / \mathrm{HPAN}$ nanomaterial, ACS Sustainable Chem. Eng., 2017, 5, 78127823.

16 B. Ramalingam, M. M. R. Khan, B. Mondal, A. B. Mandal and S. K. Das, Facile Synthesis of silver nanoparticles decorated magnetic-chitosan microsphere for efficient removal of dyes and microbial contaminants, ACS Sustainable Chem. Eng., 2015, 3, 2291-2302.

17 J. Zhu, Y. Zhang, M. Tian and J. Liu, Fabrication of a Mixed Matrix Membrane with in situ synthesized quaternized polyethylenimine nanoparticles for dye purification and reuse, ACS Sustainable Chem. Eng., 2015, 3, 690-701.

18 J. Huang, G. Arthanareeswaran and K. Zhang, Effect of silver loaded sodium zirconium phosphate (nanoAgZ) nanoparticles incorporation on PES membrane performance, Desalination, 2012, 285, 100-107.

$19 \mathrm{~J}$. Huang, H. Wang and K. Zhang, Modification of PES membrane with $\mathrm{Ag}-\mathrm{SiO}_{2}$ : Reduction of biofouling and improvement of filtration performance, Desalination, 2014, 336, 8-17.

20 Q.-F. An, W.-D. Sun, Q. Zhao, Y.-L. Ji and C.-J. Gao, Study on a novel nanofiltration membrane prepared by interfacial polymerization with zwitterionic amine monomers, $J$. Membr. Sci., 2013, 431, 171-179.

21 W. J. Lau, S. Gray, T. Matsuura, D. Emadzadeh, J. P. Chen and A. F. Ismail, A review on polyamide thin film nanocomposite (TFN) membranes: History, applications, challenges and approaches, Water Res., 2015, 80, 306-324.

22 S. M. D. Haeshin Lee, W. M. Miller and P. B. Messersmith, Mussel-Inspired surface chemistry for multifunctional coatings, Science, 2007, 318, 426-430.

23 H.-C. Yang, J. Luo, Y. Lv, P. Shen and Z.-K. Xu, Surface engineering of polymer membranes via mussel-inspired chemistry, J. Membr. Sci., 2015, 483, 42-59.

24 J. Yan, Y. Huang, Y. E. Miao, W. W. Tjiu and T. Liu, Polydopamine-coated electrospun poly(vinyl alcohol)/ poly(acrylic acid) membranes as efficient dye adsorbent with good recyclability, J. Hazard. Mater., 2015, 283, 730-739.

25 H.-C. Yang, K.-J. Liao, H. Huang, Q.-Y. Wu, L.-S. Wan and Z.-K. Xu, Mussel-inspired modification of a polymer membrane for ultra-high water permeability and oil-inwater emulsion separation, J. Mater. Chem. A, 2014, 2, 10225-10230.

26 D. R. Dreyer, D. J. Miller, B. D. Freeman, D. R. Paul and C. W. Bielawski, Perspectives on poly(dopamine), Chem. Sci., 2013, 4, 3796-3802. 
27 C. Hohme, V. Filir, C. Abetz, P. Geogopanos, N. Scharnagl and V. Abetz, Postfunctionalization of nanoporous block copolymer membranes via click Reaction on polydopamine for liquid phase separation, ACS Appl. Nano Mater., 2018, 1, 3124-3136.

28 Y. Zhao, K. Tang, H. Liu, B. Van der Bruggen, A. Sotto Díaz, J. Shen and C. Gao, An anion exchange membrane modified by alternate electro-deposition layers with enhanced monovalent selectivity, J. Membr. Sci., 2016, 520, 262-271.

29 Y. Zhao, K. Tang, Q. Liu, B. Van der Bruggen, A. S. Díaz, J. Pan, C. Gao and J. Shen, Recovery of chemically degraded polyethyleneimine by a re-modification method: prolonging the lifetime of cation exchange membranes, RSC Adv. , 2016, 6, 16548-16554.

30 J. Zhu, M. Tian, J. Hou, J. Wang, J. Lin, Y. Zhang, J. Liu and B. Van der Bruggen, Surface zwitterionic functionalized graphene oxide for a novel loose nanofiltration membrane, J. Mater. Chem. A, 2016, 4, 1980-1990.
31 B. Díez, N. Roldán, A. Martín, A. Sotto, J. A. Perdigón-Melón, J. Arsuaga and R. Rosal, Fouling and biofouling resistance of metal-doped mesostructured silica/polyethersulfone ultrafiltration membranes, J. Membr. Sci., 2017, 526, 252263.

32 E. A. Bell, T. E. Poynor, K. B. Newhart, J. Regnery, B. D. Coday and T. Y. Cath, Produced water treatment using forward osmosis membranes: Evaluation of extended-time performance and fouling, J. Membr. Sci., 2017, 525, 77-88.

33 D. Y. Zhang, J. Liu, Y. S. Shi, Y. Wang, H. F. Liu, Q. L. Hu, L. Su and J. Zhu, Antifouling polyimide membrane with surface-bound silver particles, J. Membr. Sci., 2016, 516, 83-93.

34 S. Kumar-Krishnan, E. Prokhorov, M. Hernández-Iturriaga, J. D. Mota-Morales, I. C. Sanchez and G. Luna-Bárcenas, Chitosan/silver nanocomposites: synergistic antibacterial action of silver nanoparticles and silver ions, Eur. Polym. J., 2015, 67, 242-251. 